$5-2008$

\title{
A Two-Degree-Of-Freedom Time-Optimal Solution for Hard Disk Drive Servo Problems
}

\author{
Shaohua $\mathrm{Hu}$ \\ ImaCor LLC \\ Zhiqiang Gao \\ Cleveland State University, Z.GAO@csuohio.edu
}

Follow this and additional works at: https://engagedscholarship.csuohio.edu/enece_facpub

Part of the Controls and Control Theory Commons

How does access to this work benefit you? Let us know!

Publisher's Statement

This is the accepted version of the following article: Hu, S., , \& Gao, Z. (2008). A two-degree-offreedom time-optimal solution for hard disk drive servo problems. International Journal of Adaptive Control and Signal Processing, 22(4), 388 - 401. doi:10.1002/acs.993, which has been published in final form at http://onlinelibrary.wiley.com/doi/10.1002/acs.993/abstract

\section{Original Citation}

Hu, S., , \& Gao, Z. (2008). A two-degree-of-freedom time-optimal solution for hard disk drive servo problems. International Journal of Adaptive Control and Signal Processing, 22(4), 388 - 401. doi:10.1002/ acs.993

\section{Repository Citation}

Hu, Shaohua and Gao, Zhiqiang, "A Two-Degree-Of-Freedom Time-Optimal Solution for Hard Disk Drive Servo Problems" (2008). Electrical Engineering \& Computer Science Faculty Publications. 47.

https://engagedscholarship.csuohio.edu/enece_facpub/47

This Article is brought to you for free and open access by the Electrical Engineering \& Computer Science Department at EngagedScholarship@CSU. It has been accepted for inclusion in Electrical Engineering \& Computer Science Faculty Publications by an authorized administrator of EngagedScholarship@CSU. For more information, please contact library.es@csuohio.edu. 


\title{
A two-degree-of-freedom time-optimal solution for hard disk drive servo problems
}

\author{
Shaohua $\mathrm{Hu}^{1}$ and Zhiqiang $\mathrm{Gao}^{2, *, \dagger}$ \\ ${ }^{1}$ ImaCor LLC, 50 Charles Lindberg Blvd, Suite 200, Uniondale, NY 11553, U.S.A. \\ ${ }^{2}$ Center for Advanced Control Technologies, Fenn College of Engineering, Cleveland State University, \\ Cleveland, $\mathrm{OH} 44115$, U.S.A.
}

\section{INTRODUCTION}

The computer hard disk drive (HDD) servo design plays a key role in achieving very high-speed and high-precision positioning control of magnetic heads during read/write $(\mathrm{R} / \mathrm{W})$ processes. To access the data stored in concentric tracks of a disk, two control modes are usually employed by the actuator servo: a track-seek mode and a track-following mode. The track-seek control attempts to move the heads from one track to another in minimum time, whereas the track-following servo must keep the heads at the center of a selected track as precisely as possible during a $\mathrm{R} / \mathrm{W}$ process.

\footnotetext{
*Correspondence to: Zhiqiang Gao, Center for Advanced Control Technologies, Fenn College of Engineering, Cleveland State University, Cleveland, OH 44115, U.S.A.

†E-mail: z.gao@csuohio.edu
} 
Among HDD control techniques, the mode switching control (MSC) [1] is widely employed. The MSC uses two separate controllers for the track-seek mode and the track-following mode, respectively. Classical control methods, such as the proximate time-optimal control, are adopted in the track-seek controller while lead-lag compensators or proportional-integral-derivative (PID) compensators are often used in the track-following phase.

With the rapid advance in computer hardware, the HDDs evolve toward even smaller size and higher recording density. This in turn poses bigger challenges to servo control design [2-4]. Although the existing MSC method has been used successfully in HDDs for quite some time, it is not clear such a control structure is adequate to meet the new challenges. More recently, a dualstage actuator HDD [5-7] has been employed which consists of a primary actuation stage and a secondary micro-actuator, each requiring a separate control design. Ideally, a single, well-designed, controller should be able to handle a large range of dynamic variations, such as that found in MSC and the dual-stage design. This would eliminate the need for troublesome controller switching and simplifies the controller structure and implementation. Obviously, this controller must have high performance and be highly robust for the HDD applications. The question is if such controller can be found.

In search for a unified HDD servo control algorithm, we first note that the nature of the problem is that of the well-known time-optimal control: the position error is to be reduced to zero in minimum time, given a limited motor torque. The solution in continuous time is the so-called bang-bang control, which is often impractical particularly for digital implementations. It was shown for the first time in [8] that the discrete-time closed-form solution for the time optimal control exists and it is not bang-bang. The detailed mathematical derivation was given in [9], with its properties and applications presented in [10].

The new time-optimal control solution proves to be a viable solution for the HDD servo problems in [11]. In particular, its performance and robustness were improved to such a degree over MSC that this single controller was able to replace the previous two controllers without performance degradation. This new controller, as it is applied to computer HDD problems, is denoted as the time-optimal unified servo control (TOUSC) [11]. It is a single, fixed, controller that performs the positioning control for both the track-seek and the track-following modes.

In addition to the controller design, it has been shown in the literature [12-16] that the unique problems in HDD, such as saturation, overshoot, resonant frequencies, can be addressed in the nonlinear two-degree-of-freedom (2DOF) control structure. The smooth motion profile used in the 2DOF scheme helps to prevent controller from exciting the resonant modes of HDD and the use of a feedforward term helps to reduce overshoot. The objective in this paper is to see whether the new TOUSC can be readily combined with 2DOF structure and further improve its performance.

The paper is organized as follows. The TOUSC control method is briefly outlined in Section 2 . The 2DOF-based TOUSC method is introduced in Section 3. Simulations in a 13-kTPI HDD model and robustness analysis are described in Section 4. Finally, concluding remarks are included in Section 5 .

\section{THE TOUSC CONTROL STRATEGY}

Key to the proposed new HDD servo control algorithm is the recent development in arriving at a closed-form solution for the discrete-time time-optimal control problem. This solution for a double-integral plant was first proposed as a solution to a nonlinear differentiator problem [8] but 


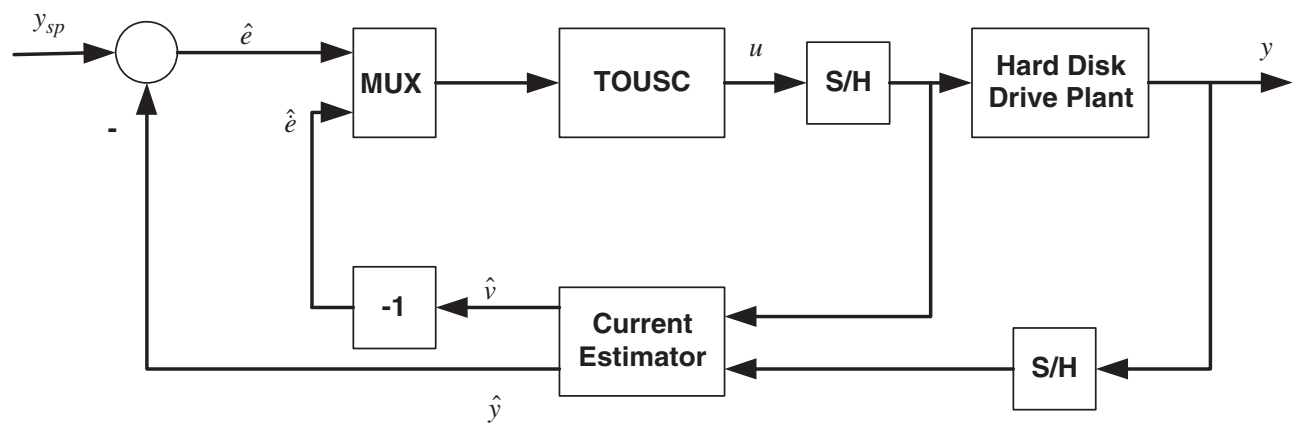

Figure 1. TOUSC control system structure.

was later shown as a general solution to servo problems $[9,10]$. It was shown [10] that such solution exhibits superior tolerance for uncertainties and disturbances. This allows relatively simple model of the plant to be used in control design and leave the controller to deal with the discrepancies between the model and the real plant. The development of TOUSC was a result of applying this solution for HDD [11].

The structure of the TOUSC is shown in Figure 1. The control system consists of two components: a state-space observer implemented in a unique digital form, known as the current estimator [17], and the TOUSC controller.

Because a high-performance motion could be quite sensitive to phase lags in the state observer, we first seek to find a digital state observer that has a small phase lag. One such observer was presented in [17]. In contrast to a conventional state observer in digital form, namely the predicted estimator, the so-called current estimator provides the estimate of the state based on the current measurements, as opposed to the previous ones. Assume that the discrete HDD plant is described by

$$
\begin{aligned}
X(k+1) & =\Phi X(k)+\Gamma u(k) \\
y(k) & =H X(k)
\end{aligned}
$$

where $u$ and $y$ are the input and output, respectively, $X$ is the state variable, and $\Phi, \Gamma$ and $H$ are constant matrices of appropriate dimensions. The current estimate $\hat{X}(k)$ is obtained as

$$
\hat{X}(k)=\bar{X}(k)+L_{c}[y(k)-H \bar{X}(k)]
$$

where $L_{c}$ is the observer gain, and $\bar{X}(k)$ is the predicted estimate based on the model in (1). That is

$$
\bar{X}(k)=\Phi \hat{X}(k-1)+\Gamma u(k-1)
$$

It can be seen in Equation (2) that the estimated state $\hat{X}(k)$ is updated using the current output of the plant, $y(k)$, instead of the previous one, $y(k-1)$. This proves to be critical in applications, where the sampling frequency is limited physically and the associated lag affects the performance of the control system.

The TOUSC block in Figure 1 is based on the discrete-time solution for time-optimal control, first proposed by Han and Yuan [8]. For the sake of simplicity, assume that the HDD can be approximated as a double-integral plant with a nominal gain, $K$. Time-optimal control for such a plant is well known in continuous time domain. The associated chattering in control signal makes it less attractive in practical use. The contribution of Han and Yuan is that they derived a 
closed-form solution in discrete-time domain which resolves the chattering issue. More details on mathematical derivation and practical applications of this solution can be found in $[9,10]$. For the sake of brevity, only the final closed-form solution is given below.

Let $R$ be the control signal saturation value, i.e. $|u(k)| \leqslant R$. With a sampling period of $h$, the TOUSC algorithm can be described as follows:

$$
\begin{gathered}
\delta=h K R, \quad \delta_{1}=h^{2} K R \\
x=\left(x_{1}, x_{2}\right)^{\mathrm{T}}=(-\hat{e},-\hat{\dot{e}})^{\mathrm{T}} \\
z_{1}=x_{1}+h x_{2}, \quad z_{2}=x_{2} \\
g(z)=\left\{\begin{array}{cc}
z_{2}-\operatorname{sign}\left(z_{1}\right) \frac{K R}{2}\left(h-\sqrt{\frac{8\left|z_{1}\right|}{K R}+h^{2}}\right), & \left|z_{1}\right|>\delta_{1} \\
z_{2}+\frac{z_{1}}{h}, & \left|z_{1}\right| \leqslant \delta_{1}
\end{array}\right. \\
u\left(z_{1}, z_{2}\right)=-R \cdot \operatorname{sat}(g(z), \delta)
\end{gathered}
$$

where

$$
\operatorname{sat}(x, \delta)= \begin{cases}\operatorname{sign}(x), & |x|>\delta \\ \frac{x}{\delta}, & |x| \leqslant \delta\end{cases}
$$

From Equations (8) and (9), it was observed that, contrary to the bang-bang control, the TOUSC is equivalent to a linear proportional control law when the error is within the region bounded by $\delta$. And this is not an approximation and it fundamentally resolves the chattering issue commonly seen in other digital implementations of the continuous time optimal control law. This is the main contribution of Han and Yuan.

We also note that, because of the aggressive nature of time optimal control laws, they tend to be sensitive to noises in the feedback. Therefore, a trade-off is often needed to balance performance and smoothness of the control signal. One such trade-off proposed in $[9,10]$ is to make the step size of TOUSC several times larger than the actual sampling periods. That is why we may replace $h$ in Equations (4) to (9) by $k_{h} * h$, where $k_{h} \geqslant 1$ is the tuning parameter. Intuitively, this expands the linear proportional control region in TOUSC and makes the control signal smoother at the cost of degradation of the performance $[9,10]$. For the HDD applications, the challenge is to make $k_{h}$ as close to unity as possible while maintaining an acceptable level of smoothness in the control signal. The $2 \mathrm{DOF}$ approach described in the following section is motivated to serve this purpose.

\section{THE 2DOF TOUSC SOLUTION}

The one-degree-of-freedom TOUSC shown in Figure 1 proves to be a very effective servo control solution, even though its derivation in $[8,9]$ assumes a double integral plant and ignores all other dynamics, such as power amplifier and resonance, as well as all nonlinearities. With inherent aggressiveness in time optimal control, TOUSC proves to be a powerful tool in dealing with dynamic uncertainties and external disturbances $[10,11]$. The endless pursuit in accuracy and bandwidth in HDD, however, demands more. The question is, if we have a fairly good plant 


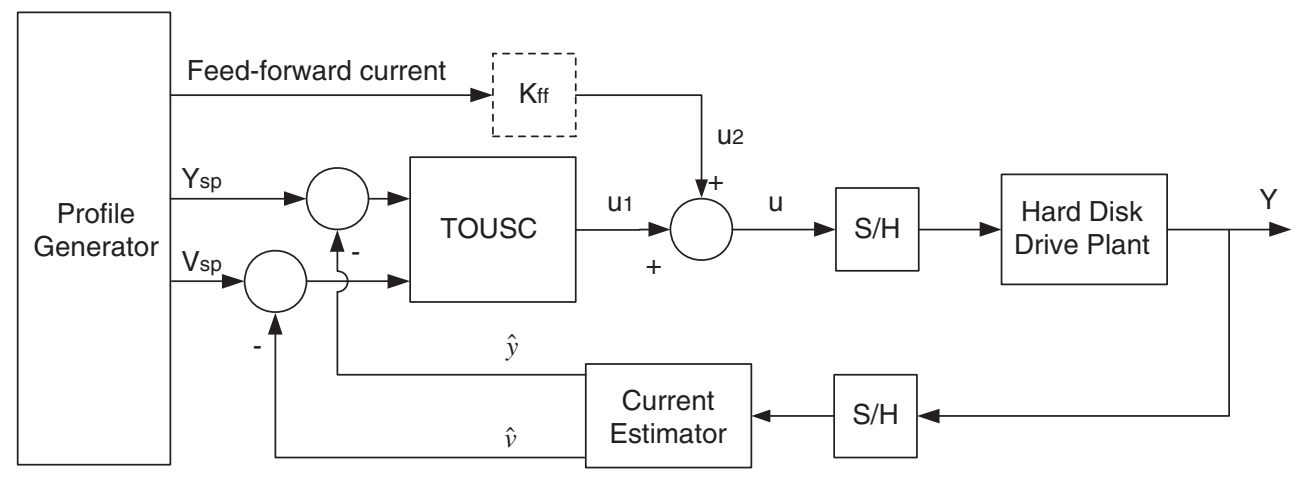

Figure 2. Structure of nonlinear 2DOF unified servo.

model, can we make TOUSC perform even better? The answer is shown in this and the next sections.

It is no secret that adding a properly constructed feedforward path will ease the burden on the feedback control and improve the overall performance. The 2DOF TOUSC solution for HDD, as shown in Figure 2, is proposed on a similar basis. Along with the TOUSC controller, the additional profile generator helps to generate a feedforward current signal bypassing the feedback loop and driving the HDD plant directly. With both feedback and feedforward controllers, this control system possesses $2 \mathrm{DOF}$ in design.

In the track-seek mode, the feedforward current dominates the control signal while the TOUSC acts as a fine-tune controller. During the track-following phase, the feedforward signal becomes negligible while the TOUSC takes the responsibility of maintaining high track-following precision. The use of the feedforward allows the TOUSC to be tuned more aggressively, which increases the positioning precision and its capability to suppress the disturbances without degrading the stability of the system. The combination of TOUSC and the feedforward control makes it feasible to replace the two separate controllers with one fixed controller, thus eliminating the need for controller switching. It is in this sense that the new control scheme is unified.

\subsection{The profile generator}

A position profile for HDD servo is used to provide the nearly fastest (time-optimal) trajectory that the HDD can possibly follow. It is also made smooth enough so that high-frequency components of the plant are not excited. A compromise is made between the speed and the smoothness in the profile design.

In order to design a profile that is physically attainable, the use of the dynamic model of the HDD plant, as shown in a simplified form in Figure 3, proves to be helpful [12]. Note that the power amplifier controller has a saturation voltage, $V_{\max }$, for its output. Based on the plant model, the structure of the profile generator is illustrated in Figure 4. Besides the double-integral motion model, it also includes the back electromotive force, the VCM model and dynamics of the power amplifier. Additionally, the DAC saturation block is needed behind the VCM model block to limit the current output and a 'non-negative' saturation block is placed to prevent the velocity from becoming negative, as seen in the figure. The DAC saturation block has $\pm R(\mathrm{~A})$ as the saturation limits while the 'non-negative' block sets zero as its lower limitation. 


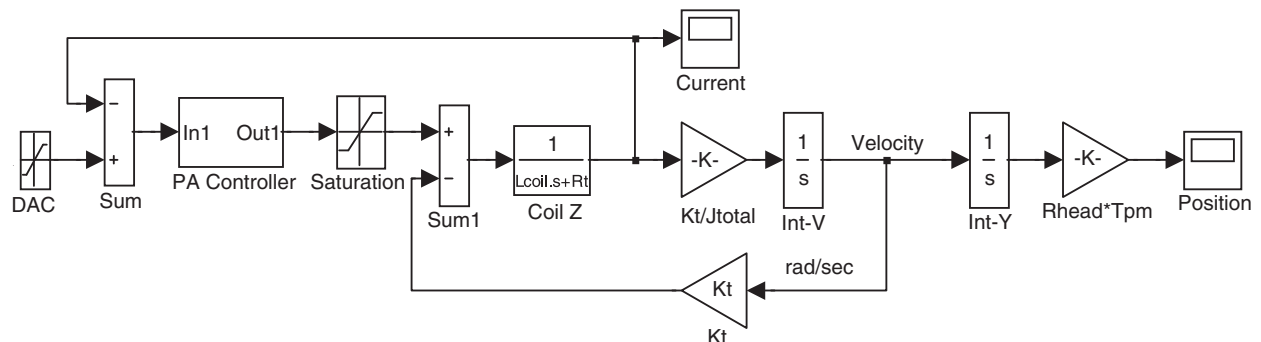

Figure 3. A simplified hard disk drive model.

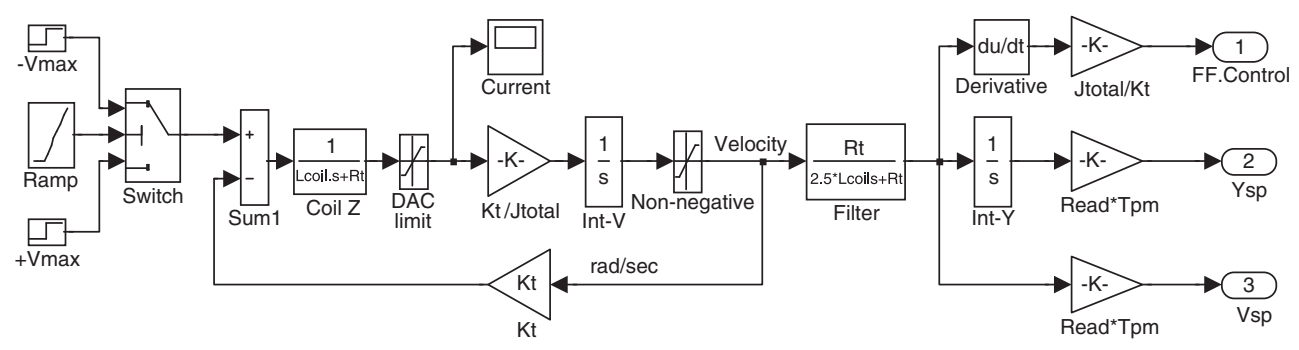

Figure 4. Structure of the profile generator.
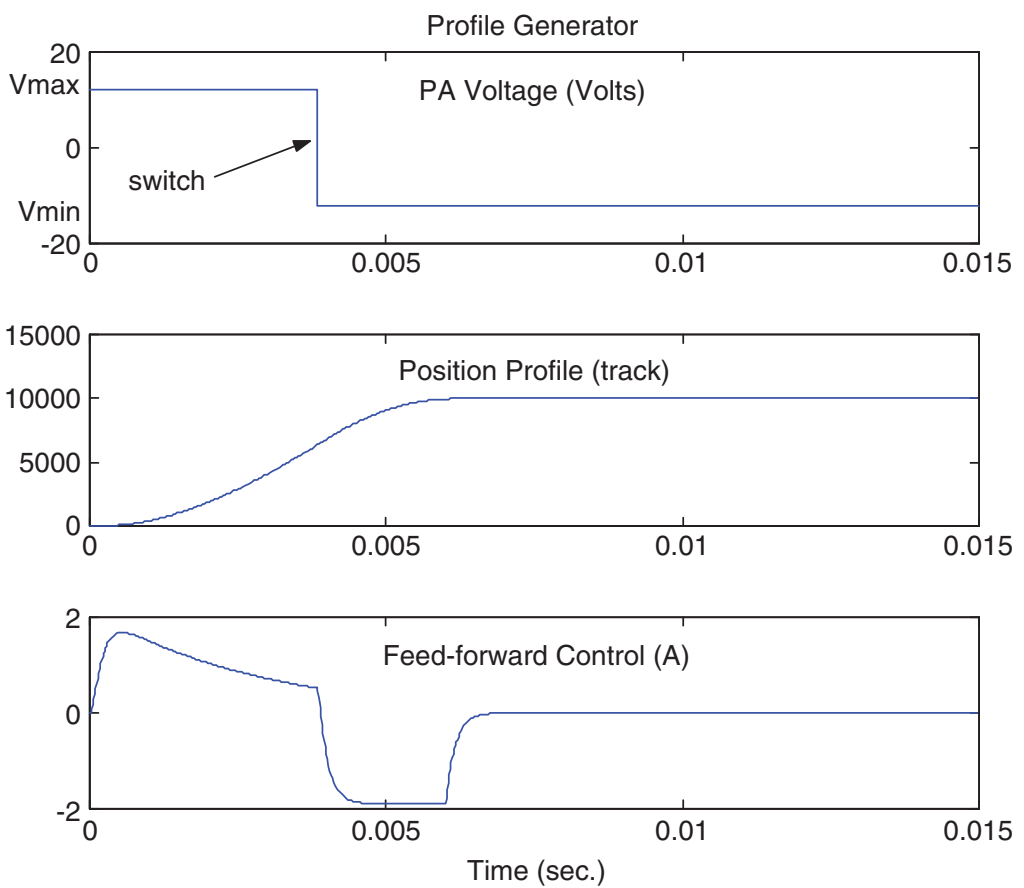

Figure 5. Inputs and outputs of the profile generator. 
To produce the fastest and attainable position profile using the plant model in the profile generator, a maximum voltage, $V_{\max }$, is first applied to the VCM model for a certain time interval, resulting in maximum acceleration. Then, the voltage is switched to the maximum negative value, $-V_{\max }$, resulting in maximum deceleration. Because of the 'non-negative' saturation block, the velocity will reduce to zero and remains the same, and the position will reach its steady-state value. The length of the time interval during which the $V_{\max }$ is applied determines the steady-state value of the position output. For example, in a 13-kTPI HDD plant described in Appendix A, it was determined by simulation that to move the position to 10000 tracks, the duration for $V_{\max }$ should be $3.829184 \times 10^{-3} \mathrm{~s}$, as shown in Figure 5 .

The simulation model is illustrated in Figure 4, where the Ramp signal triggers the Switch according to the switching time. To determine the timing of the Switch in Figure 4, off-line simulations are required to obtain a look-up table, which records the relationships between the track distance and the switch time. For each desired track distance, its switch time is obtained by reading the look-up table. To make the profile smooth and avoid exciting the high-frequency components, an additional first-order filter, $R_{t} /\left(2.5 L_{\mathrm{coil}} S+R_{t}\right)$, is applied to the velocity signal. Also, the plant model in Figure 4 is continuous and needs to be converted into its discrete form in implementation.

\subsection{Design considerations}

The profile generator provides the position reference, $Y_{\mathrm{sp}}$, and the velocity reference, $V_{\mathrm{sp}}$. The velocity reference is essential to TOUSC because the velocity tracking error is approximately

$$
\hat{\dot{e}}(t)=V_{\mathrm{sp}}(t)-\hat{v}(t)
$$

In addition, the profile generator produces the feedforward current signal.

The feedforward current signal, as shown in Figure 5 above, helps to speed up the track-seek time significantly because it avoids the lag in the feedback loop. On the other hand, it is generated from a simplified HDD model and the model mismatch with the real plant generates an equivalent of an input disturbance, which may cause a small overshoot during the transition from track seek to track following. However, the performance gain seems to outweigh the small overshoot in this case, particularly when we found that the overshoot can be managed by adjusting the feedforward gain $K_{\mathrm{ff}}$. As will be shown in the next section, the trade-off is that increasing $K_{\mathrm{ff}}$ will reduce the overshoot but slow down the response.

\section{SIMULATION AND ANALYSIS}

The proposed method is applied to a 13-kTPI HDD model in simulation in this section with a sampling rate of $15 \mathrm{kHz}$. The parameters of the disk drive model are listed in Appendix A. The $\mathrm{D} / \mathrm{A}$ output has a saturation of $\pm 1.9 \mathrm{~A}$, which corresponds to the parameter $R$ in TOUSC, and the power amplifier's maximum output voltage is $\pm 12.0 \mathrm{~V}$.

Using the zero-order hold method [17], the simplified rigid-body model of HDD, a double integrator sampled at the $15 \mathrm{kHz}$ sampling rate, as the $\Phi, \Gamma$ and $H$ matrices in (1) are obtained as

$$
\Phi=\left[\begin{array}{cc}
1 & 1.6207 \\
0 & 1
\end{array}\right], \quad \Gamma=\left[\begin{array}{l}
1.5953 \\
1.9686
\end{array}\right], \quad H=\left[\begin{array}{ll}
1 & 0
\end{array}\right]
$$


and the observer gain vector $L_{c}$ was determined as $L_{c}=\left[\begin{array}{ll}0.79 & 0.25\end{array}\right]$, first by using pole placement method and then by manually tuning later in simulation. Note that the matrices in (11) are just rough approximation of the real HDD plant.

Our simulation studies focused on three aspects: the impact of adjusting $K_{\mathrm{ff}}$, track-seek performance and track-following performance.

\subsection{Impact of the feedforward gain}

As mentioned before, the feedforward gain, $K_{\mathrm{ff}}$, is adjusted to overcome the overshoot caused by the current switch in the feedforward signal. The larger the $K_{\mathrm{ff}}$ is increased from its default value of 1 , the more action the TOUSC will produce in the track-seek process. Accordingly, smoother transition from the track seek to the track following is obtained, as shown below.

Figure 6 shows both the feedforward control signal $u_{2}$ and the TOUSC output $u_{1}$ during the track-seek process. The larger the $K_{\mathrm{ff}}$, the stronger the TOUSC control is during the seek process. The corresponding position responses are shown in Figure 7. Smoother position outputs have been achieved at the transition with relatively larger $K_{\mathrm{ff}}$. The transient performances are shown in Table I with different $K_{\mathrm{ff}}$.

It is evident that a suitable $K_{\text {ff }}$ helps to decrease the overshoot in the 2DOF-based TOUSC scheme. But, as one reviewer correctly pointed out, there is a ripple in the TOUSC signal during the transition from track-seek to track-following mode. Such ripple has high-frequency contents, which is undesirable for plants with resonant modes. Note that a very small residual oscillation still exists during the transition from track seeking to track following. This can be perhaps viewed as a possible drawback in the proposed 2DOF-based design. It calls for a judicious use of the feedforward signal. On the other hand, the fact that the residual oscillation quickly diminishes is an indication that the TOUSC controller provides good electronic damping.

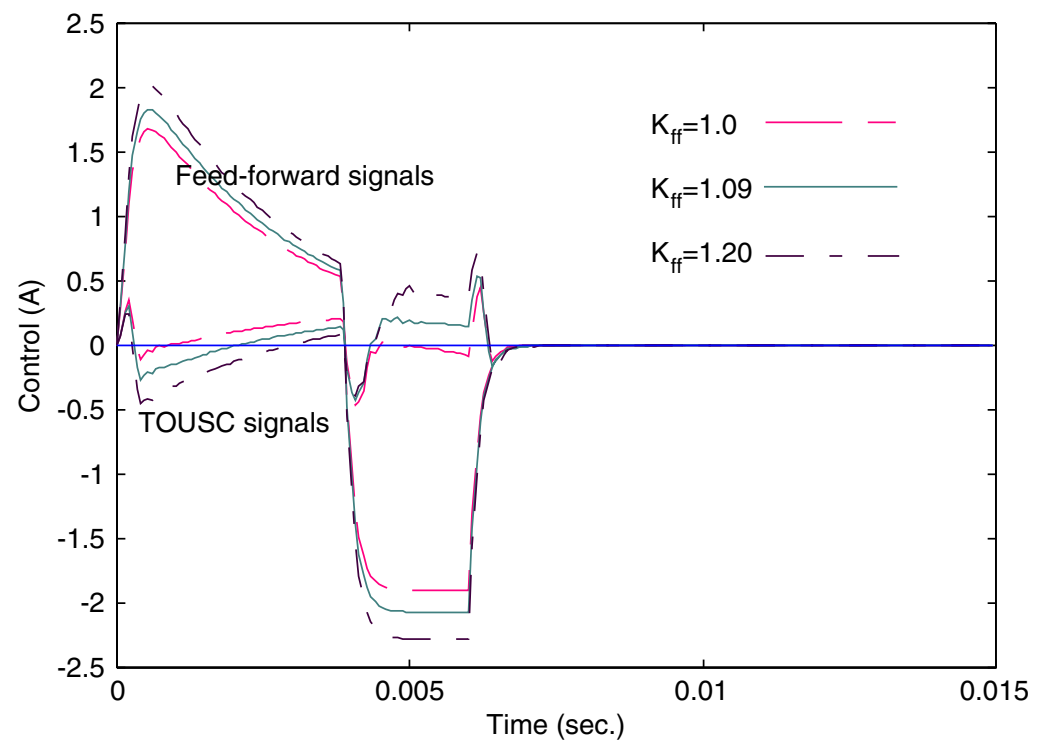

Figure 6. Control signals of the 2DOF-based TOUSC. 


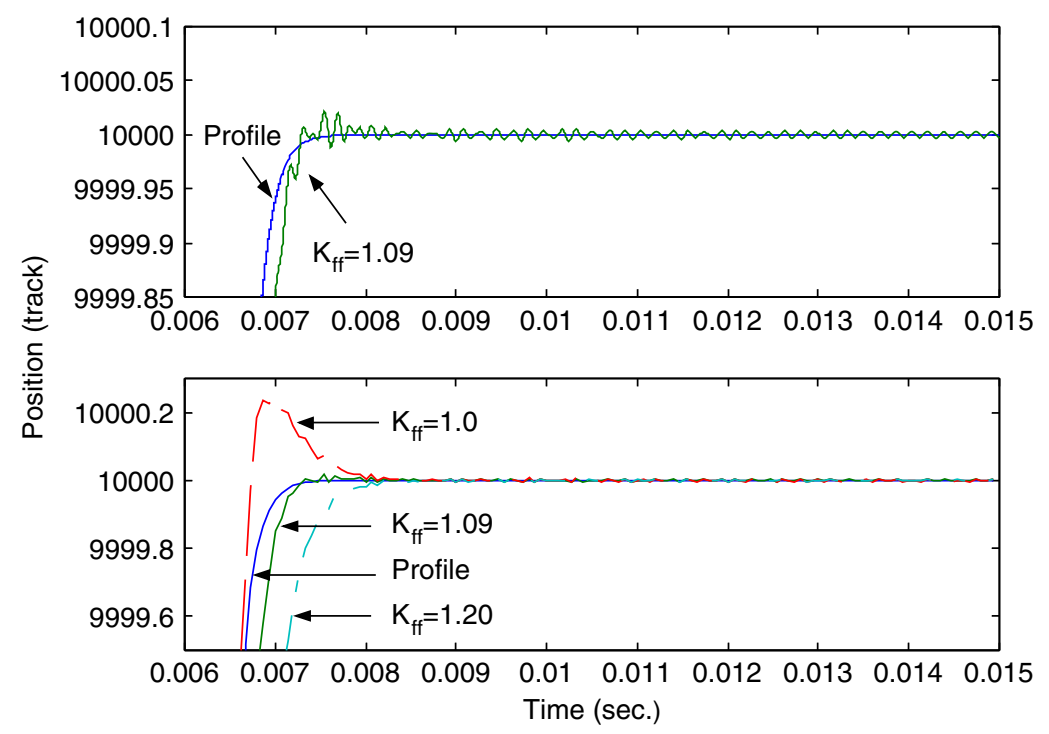

Figure 7. The position responses of the 2DOF-based TOUSC with different $K_{\mathrm{ff}}$.

Table I. Performances with different $K_{\mathrm{ff}}$.

\begin{tabular}{ccccc}
\hline$K_{\mathrm{ff}}$ & $\begin{array}{c}\text { Overshoot } \\
(\text { tracks })\end{array}$ & $\begin{array}{c}\text { Seek time } \\
(\mathrm{ms})\end{array}$ & $\begin{array}{c}\text { Settling time } \\
(0.02 \text { track })(\mathrm{ms})\end{array}$ & $\begin{array}{c}\text { Steady state } \\
\text { error (tracks) }\end{array}$ \\
\hline 1.00 & 0.23 & 7.4 & 8.0 & 0.008 \\
1.09 & 0.02 & 7.1 & 7.3 & 0.008 \\
1.20 & 0.008 & 7.6 & 8.0 & 0.005 \\
\hline
\end{tabular}

\subsection{Improvement of the track-seek performance}

To show improvement of the seek time, the proposed 2DOF-based TOUSC scheme is compared with the TOUSC method in simulation. The structures and parameters of both controllers have already been described above. For a 10000-track distance, the response curves of both cases are shown in Figure 8. Notice that $K_{\mathrm{ff}}$ is set to 1.09 and $k_{h}$ to 2.5 in the proposed method and that $k_{h}$ was 3.5 in the TOUSC.

The lower subplot in Figure 8 shows that TOUSC itself produces a quite smooth control signal and, in the proposed method, it was able to deal with the sharp change in the feedforward control current signal and smoothly enters the track-following phase. The top subplot shows that the proposed 2DOF design is able to reduce seek time significantly without incurring oscillatory response, in the presence of eight resonant modes in the simulation model.

According to the simulation, the seek time of the proposed method improves to 7.2 from $8.1 \mathrm{~ms}$ in the TOUSC case. Similarly, a series of simulation were conducted for different seek distances. The resulting curves are plotted in Figure 9. Using time-optimal profiles and feedforward control signal, the proposed method evidently produces shorter seek time than the TOUSC while still maintaining satisfactory track settling performance. 

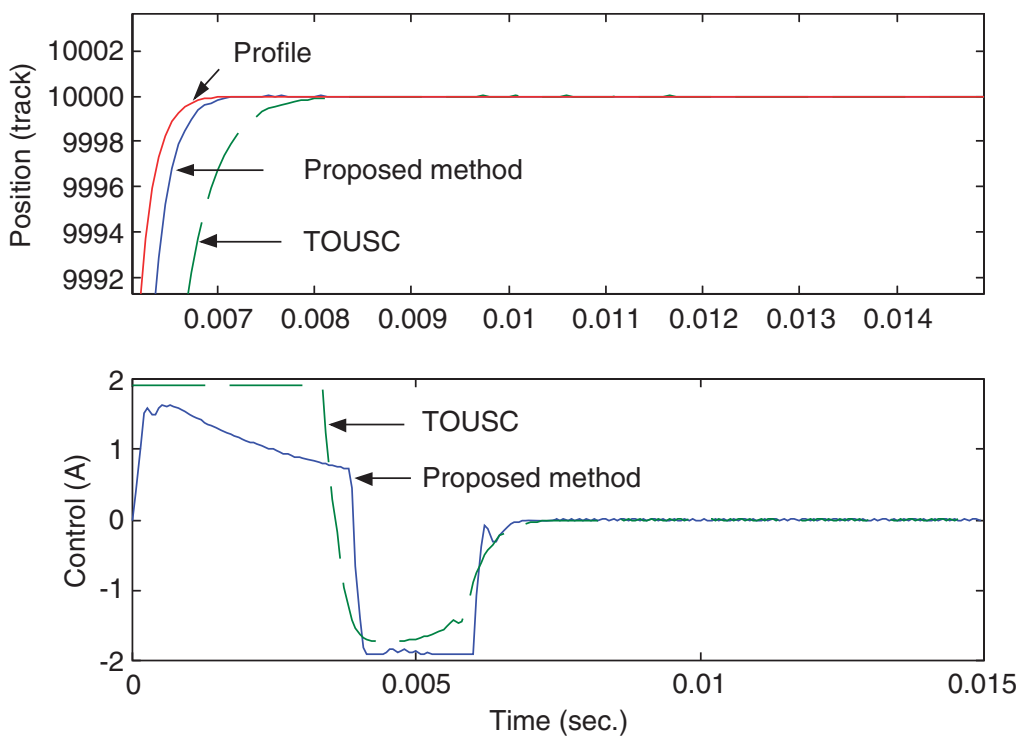

Figure 8. Simulation curves for 10000 track seek.

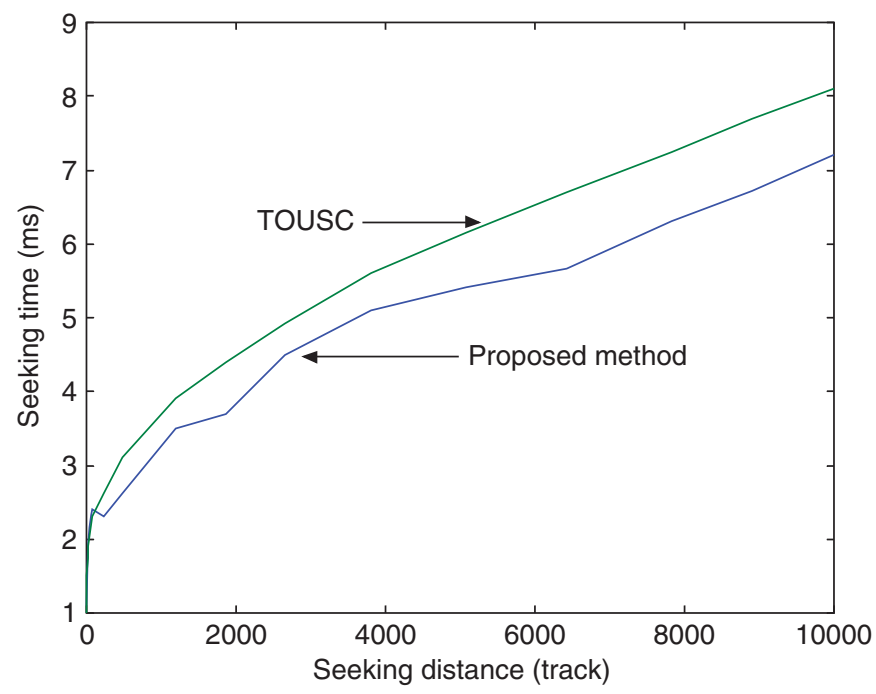

Figure 9. Comparison of seek times in simulations.

\subsection{Improvement of track-following performance}

By using the feedforward control and the smooth profile, the TOUSC is allowed to be made more aggressive. Here $k_{h}$ is reduced from 3.5 to 2.5 , which makes it closer to the ideal case of $k_{h}=1$. 

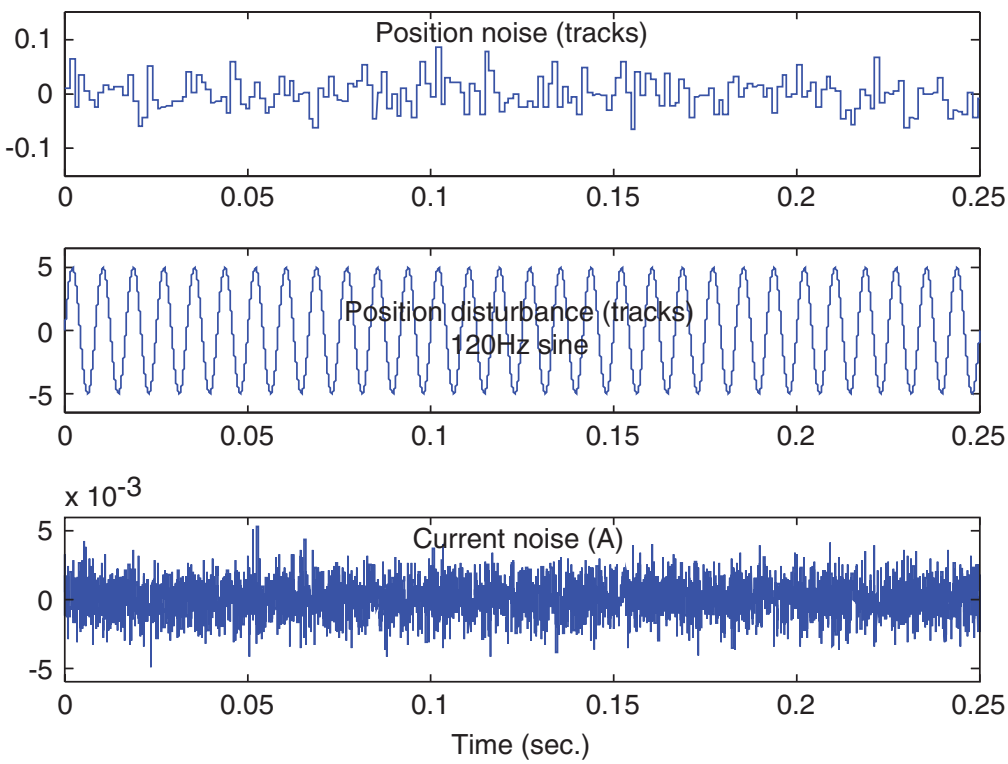

Figure 10. Position noise, vibration, and current disturbances.

Notice that a smaller $k_{h}$ leads to better robustness to external disturbances and higher position control precision.

To test the track-following performance, the outputs of feedforward signal and the profiles are set to zero and white noises are inserted as the torque and position disturbances. Three typical cases were studied in simulation on the basis of how the radial vibration disturbance was applied:

Case 1: A white position noise within \pm 0.1 track and a white current disturbance within $\pm 5 \mathrm{~mA}$ were injected to the servo systems, as shown in Figure 10.

Case 2: The simulation was repeated by injecting an additional $60 \mathrm{~Hz}$ position vibration with a 5-track amplitude.

Case 3: Same as Case 2, except the sinusoidal position disturbance is of $120 \mathrm{~Hz}$ instead of $60 \mathrm{~Hz}$ ( $120 \mathrm{~Hz}$ corresponds to $7200 \mathrm{RPM}$, a typical disk rotation speed). The position noise, vibration, and current disturbances in this case are shown in Figure 10 and the position outputs from TOUSC and 2DOF-based TOUSC are shown in Figure 11.

To compare the performance of the stand-alone TOUSC and the 2DOF-based TOUSC, the RMS error is used as the performance index, defined as

$$
e_{\mathrm{rms}}=\sqrt{\frac{\sum_{N} e(k)^{2}}{N}}
$$

where $e(k)$ is the difference between the desired position and the actual one. The simulation results are listed in Table II. It is evident that the 2DOF-based TOUSC has better robustness than the TOUSC as a track-following controller. 

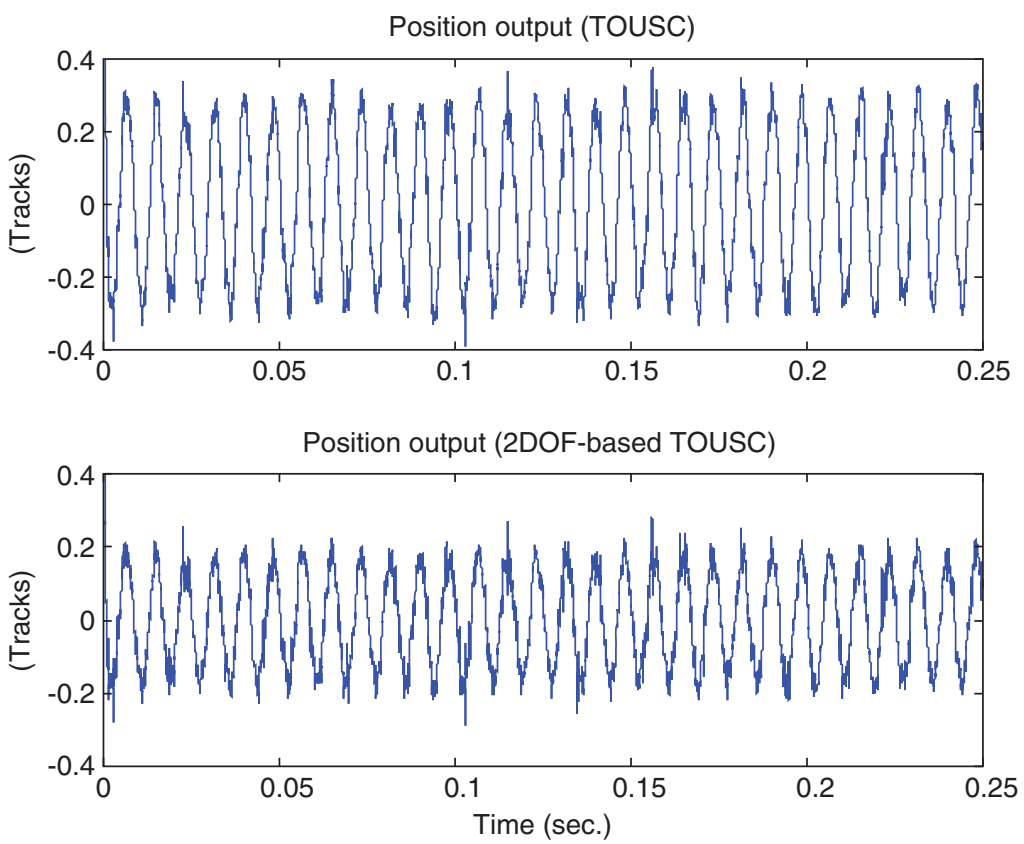

Figure 11. The track-following position outputs (case 3).

Table II. Comparison of $e_{\text {rms }}$ in track-following simulations.

\begin{tabular}{lccc}
\hline & Case 1 & Case 2 & Case 3 \\
\hline TOUSC & 0.0322 (tracks) & 0.0628 (tracks) & 0.2019 (tracks) \\
2DOF-based TOUSC & 0.0267 (tracks) & 0.0434 (tracks) & 0.1272 (tracks) \\
Improvement & $14.3 \%$ & $30.9 \%$ & $37.0 \%$ \\
\hline
\end{tabular}

\section{CONCLUDING REMARKS}

The main contribution of this paper lies in the proposed basic control structure for HDD applications and the way its components are designed and tuned. In particular,

1. Instead of relying on detailed model of HDD and employing different controllers to meet the requirements at different stages of the operation, a single feedback controller, a discrete time-optimal control solution, is employed. More importantly, the controller, along with the state estimator, is derived from the simplest model possible for a motion plant-a double integrator-ignoring all other dynamics and disturbances. Considering the lack of dependence on the accurate model, the controller performance for the HDD plant is remarkable.

2. The feedback controller is complimented by a proposed feedforward controller, taking full advantage of a more elaborate plant model when it is available. The paper demonstrates that such 2DOF structure could significantly enhance the performance over the single freedom scheme. It allows the feedback controller to be made more aggressive. Simulation on an 
industrial 13.0-kTPI HDD verifies the improvement in both track-seek and track-following modes, under various measurement noises and disturbances.

3. Both feedback and feedforward controllers are easily tuned for design trade-offs. The former is tuned by adjusting a single parameter, $k_{h}$, corresponding to the aggressiveness of the time optimal control law; and the latter is tuned by increasing the feedforward gain, $K_{\mathrm{ff}}$, until a proper balance between settling time and overshoot is attained.

For future research, both the feedback controller and state estimator can be improved by using a more elaborate plant model in their derivations. Furthermore, the small steady-state error, shown in Table I, can be reduced to zero, if necessary, by adding a small integral term to the controller that is only activated in a very small neighborhood of zero [11]. Alternatively, the extended state observer [18] can be used to zero the steady-state error without using the integral term in the controller.

Table AI. 13.0-kTPI hard disk drive model parameters.

\begin{tabular}{|c|c|c|c|}
\hline & Parameter & Description & Nominal value \\
\hline \multirow[t]{5}{*}{ Power amplifier } & $R_{\text {coil }}$ & Coil resistance & $5.9 \Omega$ \\
\hline & $L_{\text {coil }}$ & Coil inductance & $0.368 \mathrm{mH}$ \\
\hline & $K_{\mathrm{pa}}$ & Control gain & $1.0 \times 10^{6}$ \\
\hline & $e_{\max }$ & Saturated voltage & $12.0 \mathrm{~V}$ \\
\hline & Slewrate & DAC rate limit & $10000 \mathrm{~A} / \mathrm{s}$ \\
\hline \multirow[t]{5}{*}{ Actuator } & $J_{\text {total }}$ & Moving inertia & $2.54 \times 10^{-6} \mathrm{~kg} \mathrm{~m}^{2}$ \\
\hline & $K_{\mathrm{t}}$ & Torque constant & $0.075 \mathrm{~N} \mathrm{~m} / \mathrm{A}$ \\
\hline & $R_{\text {head }}$ & Head radius & $1.9 \mathrm{in}$ \\
\hline & TPI & Tracks density & $13000 \mathrm{TPI}$ \\
\hline & Curclip & DAC saturation & $\pm 1.9 \mathrm{~A}$ \\
\hline \multirow[t]{24}{*}{ Resonance } & $f_{1}$ & 1st frequency & $4500 \mathrm{~Hz}$ \\
\hline & $f_{2}$ & 2nd frequency & $5400 \mathrm{~Hz}$ \\
\hline & $f_{3}$ & 3rd frequency & $5550 \mathrm{~Hz}$ \\
\hline & $f_{4}$ & 4 th frequency & $5670 \mathrm{~Hz}$ \\
\hline & $f_{5}$ & 5 th frequency & $7300 \mathrm{~Hz}$ \\
\hline & $f_{6}$ & 6th frequency & $7450 \mathrm{~Hz}$ \\
\hline & $f_{7}$ & 7 th frequency & $8000 \mathrm{~Hz}$ \\
\hline & $f_{8}$ & 8th frequency & $9650 \mathrm{~Hz}$ \\
\hline & $b_{1}$ & 1 st coupling coefficient & 1700 \\
\hline & $b_{2}$ & 2nd coupling coefficient & 260 \\
\hline & $b_{3}$ & 3rd coupling coefficient & 300 \\
\hline & $b_{4}$ & 4th coupling coefficient & 45 \\
\hline & $b_{5}$ & 5th coupling coefficient & 100 \\
\hline & $b_{6}$ & 6th coupling coefficient & 105 \\
\hline & $b_{7}$ & 7th coupling coefficient & 105 \\
\hline & $b_{8}$ & 8th coupling coefficient & 455 \\
\hline & $\xi_{1}^{\circ}$ & 1st damping ratio & 0.018 \\
\hline & $\xi_{2}$ & 2nd damping ratio & 0.025 \\
\hline & $\xi_{3}$ & 3rd damping ratio & 0.025 \\
\hline & $\xi_{4}$ & 4th damping ratio & 0.001 \\
\hline & $\xi_{5}$ & 5th damping ratio & 0.010 \\
\hline & $\xi_{6}$ & 6th damping ratio & 0.010 \\
\hline & $\xi_{7}$ & 7 th damping ratio & 0.010 \\
\hline & $\xi_{8}$ & 8th damping ratio & 0.013 \\
\hline
\end{tabular}




\section{APPENDIX A}

The model of the VCM actuator of an industrial 13.0-kTPI hard disk drive is described by

$$
G(s)=\frac{Y(s)}{I_{c}(s)}=\frac{K_{t} R_{\text {head }}}{J_{\text {total }} s^{2}}+{ }_{i=1}^{8} \frac{b_{i}}{s^{2}+2{ }_{i} \omega_{i} s+\omega_{i}^{2}}
$$

where $i_{c}$ is the current command (in ampere) and $y$ is the position output (in meter). The model parameters are listed in Table AI.

\section{ACKNOWLEDGEMENTS}

The authors would like to thank Dr Lin Yang for his encouragement and assistance in this research. They also would like to thank the anonymous reviewers for their helpful suggestions.

\section{REFERENCES}

1. Yamaguchi T, Shishida K. A mode-switching controller with initial value compensation for hard disk drive servo control. Control Engineering Practice 1997; 5(11):1525-1532.

2. Venkataramanan V, Peng K, Chen BM, Lee TH. Discrete-time composite nonlinear feedback control with an application in design of a hard disk drive servo system. IEEE Transactions on Control Systems Technology 2003; 11(1):16-23.

3. Horowitz R, Li B. Adaptive track-following servos for disk file actuators. IEEE Transactions on Magnetics 1996; 32(3):1779-1786. DOI: $10.1109 / 20.492865$.

4. Oswald RK. Design of a disk file head-positioning servo. IBM Journal of Research and Development 1974; 18:506-512.

5. Kim YH, Lee SH. An approach to dual-stage servo design in computer disk drives. IEEE Transactions on Control Systems Technology 2004; 12(1):12-20.

6. Evans RB, Griesbach JS, Messner WC. Piezoelectric microactuator for dual stage control. IEEE Transactions on Magnetics 1999; 35(7):977-982.

7. Stevens F, DeLillis J. Keeping heads on track with dual-stage actuators. Data Storage 2000; 7:39-44.

8. Han J, Yuan L. The discrete form of tracking-differentiator. Journal of Systems Science and Mathematical Sciences (Chinese) 1999; 19(3):268-273.

9. Gao Z. On discrete time optimal control: a closed-form solution. Proceedings of the 2004 American Control Conference, vol. 1, Boston, MA, 2004; 52-58.

10. Gao Z, Hu S. On properties and applications of a new form of discrete time optimal control law. Proceedings of the 39th IEEE IAS Annual Meeting, vol. 3, Seattle, WA, 2004; 1511-1518.

11. Hu S. On high performance servo control algorithms for hard disk drive. Doctoral Dissertation, Cleveland State University, 2001.

12. Yi L, Tomizuka M. Two degree-of-freedom control with adaptive robust control for hard disk servo systems. IEEE/ASME Transactions on Mechatronics 1999; 1(4):17-24.

13. Chung CC, Seo CW, Lee SH. Two degree-of-freedom dual stage actuator controller design for hard disk drive. IEEE Transactions on Magnetics 2000; 36(5):2255-2257.

14. Takakura S. Design of a tracking system using $n$-delay two-degree-of-freedom control and its application to hard disk drives. Proceedings of the IEEE International Conference on Control Applications, vol. 1, Kohala Coast, HI, 1999; 170-175.

15. Jinzenji A, Sasamoto T, Aikawa K, Yoshida S, Aruga K. Acceleration feedforward control against rotational disturbance in hard disk drives. IEEE Transactions on Magnetics 2001; 37(2):888-893.

16. Huang Y, Messner WC, Steele J. Feed-forward algorithms for time-optimal setting of hard disk drive servo systems. Proceedings of the 23rd International Conference on Industrial Electronics and Control Instrumentation, vol. 1, New Orleans, LA, 1997; 52-57.

17. Franklin GF, Powell JD, Workman ML. Digital Control of Dynamic Systems (3rd edn). Addison-Wesley: Boston, MA, 1998.

18. Zheng Q, Gao Z. Motion control design optimization: problem and solutions. International Journal of Intelligent Control and Systems 2005; 10(4):269-276. 